\title{
Multicenter Tract-Based Analysis of Microstructural Lesions within the Alzheimer's Disease Spectrum: Association with Amyloid Pathology and Diagnostic Usefulness
}

Stefan J. Teipel ${ }^{\mathrm{a}, \mathrm{b}, *}$, Jan O. Kuper-Smith ${ }^{\mathrm{a}}$, Claudia Bartels ${ }^{\mathrm{c}, \mathrm{d}}$, Frederic Brosseron ${ }^{\mathrm{e}, \mathrm{f}}$, Martina Buchmann ${ }^{\mathrm{g}, \mathrm{h}}$, Katharina Buerger ${ }^{\mathrm{i}, \mathrm{j}}$, Cihan Catak $^{\mathrm{j}}$, Daniel Janowitz ${ }^{\mathrm{j}}$, Peter Dechent ${ }^{\mathrm{k}}$, Laura Dobisch $^{1}$, Birgit Ertl-Wagner ${ }^{\mathrm{m}, \mathrm{x}}$, Klaus Fließbach ${ }^{\mathrm{e}, \mathrm{f}}$, John-Dylan Haynes ${ }^{\mathrm{n}}$, Michael T. Heneka ${ }^{\mathrm{e}, \mathrm{f}}$, Ingo Kilimann ${ }^{\mathrm{a}, \mathrm{b}}$, Christoph Laske ${ }^{\mathrm{g}, \mathrm{h}}$, Siyao Li $^{\mathrm{o}}$, Felix Menne ${ }^{\mathrm{o}}$, Coraline D. Metzger ${ }^{1, p, q}$, Josef Priller ${ }^{\mathrm{r}, \mathrm{s}}$, Verena Pross ${ }^{\mathrm{t}}$, Alfredo Ramirez ${ }^{\mathrm{u}}$, Klaus Scheffler ${ }^{\mathrm{v}}$, Anja Schneider ${ }^{e, f}$, Annika Spottkee,w , Eike J. Spruth ${ }^{\mathrm{r}, \mathrm{s}}$, Michael Wagnere,f, Jens Wiltfang, ${ }^{\mathrm{e}, \mathrm{d}}$, Steffen Wolfsgruber ${ }^{\mathrm{e}}$, Emrah Düzel ${ }^{\mathrm{l}, \mathrm{p}}$, Frank Jessen ${ }^{\mathrm{e}, \mathrm{u}}$, Martin Dyrba ${ }^{\mathrm{b}}$ and the DELCODE study group ${ }^{a}$ Department of Psychosomatic Medicine, University of Rostock, Rostock, Germany

${ }^{\mathrm{b}}$ German Center for Neurodegenerative Diseases (DZNE), Rostock, Germany

${ }^{\mathrm{c}}$ German Center for Neurodegenerative Diseases (DZNE), Goettingen, Germany

${ }^{\mathrm{d}}$ Department of Psychiatry and Psychotherapy, University Medical Center Goettingen, University of Goettingen, Goettingen, Germany

${ }^{\mathrm{e}}$ German Center for Neurodegenerative Diseases (DZNE), Bonn, Germany

${ }^{\mathrm{f}}$ Department for Neurodegenerative Diseases and Geriatric Psychiatry, University Hospital Bonn, Bonn, Germany

${ }^{\mathrm{g}}$ German Center for Neurodegenerative Diseases (DZNE), Tübingen, Germany

${ }^{\mathrm{h}}$ Section for Dementia Research, Hertie Institute for Clinical Brain Research and Department of Psychiatry and Psychotherapy, University of Tübingen, Tübingen, Germany

${ }^{\mathrm{i}}$ German Center for Neurodegenerative Diseases (DZNE, Munich), Munich, Germany

${ }^{\mathrm{j}}$ Institute for Stroke and Dementia Research (ISD), University Hospital, LMU Munich, Munich, Germany

${ }^{\mathrm{k}}$ MR-Research in Neurology and Psychiatry, Georg-August-University Göttingen, Göttingen, Germany

${ }^{1}$ German Center for Neurodegenerative Diseases (DZNE), Magdeburg, Germany

${ }^{\mathrm{m}}$ Institute for Clinical Radiology, Ludwig-Maximilians-University, Munich, Germany

${ }^{\mathrm{n}}$ Bernstein Center for Computational Neuroscience, Charité - Universitätsmedizin Berlin, Berlin, Germany

${ }^{\mathrm{o}}$ Institute of Psychiatry and Psychotherapy, Charité - Universitätsmedizin Berlin, Berlin, Germany

${ }^{\mathrm{P}}$ Institute of Cognitive Neurology and Dementia Research (IKND), Otto-von-Guericke University,

Magdeburg, Germany

${ }^{\mathrm{q}}$ Department of Psychiatry and Psychotherapy, Otto-von-Guericke University, Magdeburg, Germany

\footnotetext{
${ }^{*}$ Correspondence to: Stefan J. Teipel, MD, Department of Psychosomatic Medicine, University Medicine Rostock, and DZNE, Gehlsheimer Str. 20, 18147 Rostock, Germany. Tel.: +49 381
} 
${ }^{\mathrm{r}}$ Department of Psychiatry and Psychotherapy, Charité - Universitätsmedizin Berlin, Berlin, Germany

${ }^{\mathrm{s}}$ German Center for Neurodegenerative Diseases (DZNE), Berlin, Germany

${ }^{\mathrm{t}}$ Study Center Bonn, Medical Faculty, Bonn, Germany

${ }^{\mathrm{u}}$ Department of Psychiatry, University of Cologne, Cologne, Germany

${ }^{\mathrm{v}}$ Department for Biomedical Magnetic Resonance, University of Tübingen, Tübingen, Germany

${ }^{\mathrm{w}}$ Department of Neurology, University of Bonn, Bonn, Germany

${ }^{\mathrm{x}}$ Division of Neuroradiology, Department of Medical Imaging, The Hospital for Sick Children, Toronto, Canada

Handling Associate Editor: Juan Helen Zhou

Accepted 30 August 2019

\begin{abstract}
Diffusion changes as determined by diffusion tensor imaging are potential indicators of microstructural lesions in people with mild cognitive impairment (MCI), prodromal Alzheimer's disease (AD), and AD dementia. Here we extended the scope of analysis toward subjective cognitive complaints as a pre-MCI at risk stage of AD. In a cohort of 271 participants of the prospective DELCODE study, including 93 healthy controls and 98 subjective cognitive decline (SCD), $45 \mathrm{MCI}$, and $35 \mathrm{AD}$ dementia cases, we found reductions of fiber tract integrity in limbic and association fiber tracts in $\mathrm{MCI}$ and $\mathrm{AD}$ dementia compared with controls in a tract-based analysis $(p<0.05$, family wise error corrected). In contrast, people with SCD showed spatially restricted white matter alterations only for the mode of anisotropy and only at an uncorrected level of significance. DTI parameters yielded a high cross-validated diagnostic accuracy of almost $80 \%$ for the clinical diagnosis of $\mathrm{MCI}$ and the discrimination of $\mathrm{A} \beta$ positive MCI cases from $\mathrm{A} \beta$ negative controls. In contrast, DTI parameters reached only random level accuracy for the discrimination between $A \beta$ positive SCD and control cases from A $\beta$ negative controls. These findings suggest that in prodromal stages of $\mathrm{AD}$, such as in $\mathrm{A} \beta$ positive $\mathrm{MCI}$, multicenter $\mathrm{DTI}$ with prospectively harmonized acquisition parameters yields diagnostic accuracy meeting the criteria for a useful biomarker. In contrast, automated tractbased analysis of DTI parameters is not useful for the identification of preclinical AD, including A $\beta$ positive SCD and control cases.
\end{abstract}

Keywords: amyloid, anisotropy, cerebral white matter, cognition, diagnosis, diffusion tensor imaging, mild cognitive impairment, subjective cognitive decline

\section{INTRODUCTION}

Microstructural lesions of associative fiber tracts in Alzheimer's disease (AD) likely result from primary cell loss in grey matter regions but also reflect primary white matter pathology such as myelin break down (for review, see [1]). Diffusion tensor imaging (DTI) can usefully be employed for the in vivo detection of such lesions [2, 3] showing moderate to high diagnostic accuracy in mild cognitive impairment (MCI) as a prodromal stage of AD compared with healthy controls in monocenter studies $[4,5]$. In addition, a multicenter study from retrospectively pooled DTI data [6] suggested a high diagnostic utility (about $77 \%$ cross-validated accuracy) for the discrimination between amyloid positive people with mild cognitive impairment (MCI) and healthy controls [7]. In this study, DTI was superior to volumetric measures despite high vulnerability of the DTI parameters to multicenter variability [8]. This finding was very interesting because a promising biomarker for $\mathrm{AD}$ should also prove itself in a multicenter setting [9], which is much closer to the future application in routine care than a monocenter study.

Subjective cognitive decline (SCD) is a clinical atrisk stage for MCI and dementia [10]. SCD cases have a two-fold increased risk to develop dementia and a six-fold increased risk to develop MCI over on average four years of clinical follow-up compared with cognitively normal people without subjective cognitive complaints [11]. Several studies found significant differences in DTI parameters, such as fractional anisotropy or mean diffusivity, in SCD cases compared with controls [12-15]. Studies on the diagnostic utility of DTI markers, however, for the discrimination between SCD cases and controls are still scarce. One recent monocenter study reported an area under the ROC curve of $78 \%$ for the discrimination between 
20 SCD cases and 22 controls, but only in the training sample, i.e., without cross-validation [16].

Since reliable acquisition across different sites is an important prerequisite for a potentially useful biomarker, here we tested group differences and diagnostic usefulness of microstructural lesion markers across the entire AD spectrum from a prospective multicenter DTI acquisition with harmonized acquisition parameters. The cohort spans from cognitively healthy controls through SCD and MCI to AD dementia. The current analysis focused on two endpoints, discrimination of the clinical diagnoses SCD and MCI from healthy controls and the discrimination of amyloid positive SCD and MCI cases, representing preclinical or prodromal stages of $\mathrm{AD}$ [17], from amyloid negative controls based on cerebrospinal fluid (CSF) amyloid- $\beta(\mathrm{A} \beta)$. We used tract-based statistics that was found in a previous multicenter reliability study to be less prone to multicenter effects than voxel-based analysis [8]. The motivation of our study was that if found useful in a prospective cohort tract-based statistics of microstructural lesion markers may be employed for risk stratification of study participants in future clinical trials.

\section{MATERIALS AND METHODS}

\section{Participants}

Data used in this study came from baseline data of the DELCODE (DZNE Longitudinal Cognitive Impairment and Dementia) study, an ongoing observational longitudinal memory clinic-based multicenter study in Germany [18]. A total of 282 participants from nine centers were included in this study. Two cases were excluded due to neuroimaging issues. One scan deviated in the number of slices (47 instead of 72) and slice spacing (3.5 mm instead of $2 \mathrm{~mm}$ ). Another participant had a small falx meningioma and was excluded to avoid problems with the image processing algorithms. This left us with a final number of 280 participants from nine sites. DELCODE exclusion criteria dictate that no participant should have past or present unstable medical conditions, major psychiatric disorders, including a current major depressive episode, or neurologic diseases that are not AD [18]. The basis of these exclusion criteria was provided by a clinical assessment of cognitive status, which included the Geriatric Depression Scale (GDS) [19], and an extensive neuropsychological testing battery [18].
The sample included people with SCD who were cognitively unimpaired and stated to have decline in cognitive functioning unrelated to an event or condition explaining the cognitive deficits according to research criteria [10], MCI who met National Institute on Aging - Alzheimer's Association (NIA-AA) workgroup core criteria for MCI [20], AD dementia who met the NIA-AA probable AD dementia criteria [21], and cognitively normal controls who never reported SCD and had no history of neurological or psychiatric disease or any sign of cognitive decline.

Written informed consent was provided by all participants, or their representatives. The study was approved by local ethics committees at each of the participating centers, and has been conducted in accord with the Helsinki Declaration of 1975.

\section{Imaging data acquisition}

Imaging data at the nine different DZNE sites were obtained using Siemens 3.0 Tesla MRI scanners (three Verio, three TimTrio, one Prisma, two Skyra) using the same acquisition parameters and instructions. An axial diffusion sequence was measured based on a single shot echo planar imaging sequence (acquisition time: $14 \mathrm{~min} 45 \mathrm{~s}$, field of view: 240x240 mm, isotropic voxel size: $2 \mathrm{~mm}$, repetition time: $12100 \mathrm{~ms}$, echo time: $88 \mathrm{~ms}$, flip angle: $90^{\circ}$, number of gradients: 60 , b-values: $700 \mathrm{~s} / \mathrm{mm}^{2}$ and $1000 \mathrm{~s} / \mathrm{mm}^{2}$, number of slices: 72 , parallel imaging acceleration factor: 2). High-resolution T1weighted anatomical images were obtained using a sagittal magnetization-prepared rapid gradient echo (MPRAGE) sequence (acquisition time: $5 \mathrm{~min} 8 \mathrm{~s}$, field of view: $256 \times 256 \mathrm{~mm}$, isotropic voxel size: $1 \mathrm{~mm}$, echo time: $4.37 \mathrm{~ms}$, flip angle: $7^{\circ}$, repetition time: $2500 \mathrm{~ms}$, number of slices: 192, parallel imaging acceleration factor: 2). To ensure high image quality, all scans had to pass a semi-automated check for SOP conformity and scan quality during data collection so that protocol deviations were reported to the study sites promptly, in order to allow the sites to adjust their acquisition. Additionally, all scans were visually inspected and controlled for 1) proper alignment of the field-of-view to cover the whole brain, 2) screened for severe imaging artifacts (e.g., aliasing/ghosting, strong noise/motion or susceptibility artifacts from metallic dental fillings), and 3) checked for incidental findings such as old strokes or meningiomas. 


\section{Biomaterial sampling}

Biomaterial sampling included CSF in those participants, who consented. Trained study assistants performed the collection, processing and storage of the samples up to the shipment to the central biorepository of the DZNE according to SOP. After the centrifugation CSF was aliquoted and stored at $-80^{\circ} \mathrm{C}$.

\section{Image processing}

Due to varying degrees of atrophy between participants, accurately registering white matter (WM) into a standard space is problematic for whole-brain deformation approaches [22], especially when considering smaller anatomical structures such as the fornix [23, 24]. In addition, in a previous clinical and physical phantom study, tract-based statistics (TBSS) was found less prone to scanner effects than voxelbased analysis [8]. Therefore, we used TBSS [25] in FSL 5.0.9 for DTI data analysis. First, diffusion scans were corrected for distortions using a gradient-echo field map and the T1-weighted scans by applying fsl_prepare_fieldmap and epi_reg commands. After head motion and eddy current correction [26] using eddy_correct with spline interpolation, surrounding skull matter was removed from the non-diffusionweighted images using FSL's brain extraction tool (bet2) [27] and diffusion tensor models were fitted using FSL's dtifit command to derive voxel-wise FA, MD, and mode of anisotropy values. The next steps involved aligning all subjects' FA images in a voxelwise nonlinear registration to MNI152 reference space, and creating a mean FA average from these transformed FA images. We then created a custom mean FA skeleton, which was thresholded at 0.2 in order to exclude more peripheral tracts with lower inter-subject correspondence. The individual participants' FA maps were then projected onto the skeleton by assigning the maximum FA value in perpendicular tract direction to the skeleton voxel at each point of the skeleton. This projection information was subsequently applied to mode of anisotropy and MD maps as well.

For comparison, we used classification accuracy based on hippocampus volume, an established biomarker of AD [28]. For hippocampus volumetry, we used the harmonized hippocampus segmentation protocol, an internationally driven effort under the auspices of the Alzheimer's Association [29]. Further details can be found on the project's website (http://www.hippocampalprotocol.net/SOPs/index.php). More recently, the manual hippocampus labels were integrated into an automated volumetry pipeline to ease processing of larger numbers of cases [30]. A high correspondence between manual and automated segmented hippocampi based on the harmonized protocol was shown in 135 MRI scans that were measured using both manual segmentation and automated segmentation. Following this automated processing pipeline, the T1-weighed MPRAGE images were normalized to the MNI reference template from CAT12 using SPM12 new segment and the Diffeomorphic Anatomical Registration Through Exponentiated Lie algebra (DARTEL) algorithm [31]. Subsequently, hippocampus volume was automatically computed from all voxels within the harmonized hippocampus mask regions of the normalized and modulated grey matter maps [30]. The raw volume estimates were proportionally scaled to total intracranial volume to adjust for head size.

\section{CSF AD biomarker assessment}

CSF $A \beta_{42}$ and $A \beta_{40}$ levels were determined using commercially available kits according to vendor specifications (V-PLEX A $\beta$ Peptide Panel 1 (6E10) Kit). Cut-offs for normal and abnormal concentrations of $A \beta_{42}(<496 \mathrm{pg} / \mathrm{ml})$, and of the ratio $\mathrm{A} \beta_{42} / \mathrm{A} \beta_{40}(<0.09)$ were derived from the literature, which applied the respective assays [32]. Correspondingly, cases below the cut-off of 0.09 for the ratio $A \beta_{42} / A \beta_{40}$ were designated amyloid positive, cases above this cutoff as amyloid negative.

\section{Data analysis}

\section{Demographic data}

Participants' demographic information was analyzed using appropriate tests as needed: gender distribution was assessed using $\chi^{2}$-test, while differences in age, years of education, and MMSE scores were assessed with ANOVA models.

\section{Voxelwise TBSS analysis}

Voxelwise cross-subject comparisons were performed with the control group as reference; i.e., controls versus SCD, controls versus MCI, and controls versus AD. The models included age, sex, and scanner as covariates. For the main results, we applied a significance threshold of $p<0.05$, familywise error (FWE) corrected, and for statistical trends 
$p<0.01$ uncorrected for multiple comparisons. Statistical differences were estimated by a permutation test approach with 5000 random permutations defining a null distribution of regression parameters.

We used variance component analysis to assess the vulnerability of tract-based DTI parameters to multicenter effects. For this, we extracted the subject level TBSS clusters using the FSL function "fslmeants" averaging the values of all significant voxels for the group comparisons of SCD cases versus controls and of MCI cases versus controls, respectively. This resulted in a scalar value for each individual for each comparison and each DTI parameter. We determined a random effects model in R using library "Imer" with the averaged cluster values as dependent variable and scanner as random effects variable. We then extracted the amount of variance attributable to the random effect of scanner divided by the total amount of variance, providing an estimate of the variance component for scanner for a given comparison and DTI parameter.

\section{Elastic net regression}

We calculated group discrimination using a penalized logistic regression model with an elastic net penalty, using the R package glmnet (available at http://cran.r-project.org/web/packages/glmnet/index. $\mathrm{html})$. In an elastic net regression, two penalty terms are added as an extension of the residual sum of squares minimization of traditional linear regression models to account for high collinearity of regression features [33]. A detailed description of this method as applied to multicenter imaging data can be found in [34]. Mean values were extracted from the normalized and skeletonized FA, mode of anisotropy and MD maps using the JHU-ICBM DTI atlas containing labels for 48 major white matter tracts [35]. The vectors of all three DTI indices were concatenated and entered as predictors in the logistic regression models. To assess diagnostic accuracy, we followed a stringent cross-validation procedure based on 100 times repeated $2 / 3$ by $1 / 3$ cross-validation. Estimations of the area under the receiver-operating-characteristics curve (AUC) were made for each of the iterations from the test sample. Due to currently missing extended inference techniques for elastic-net models, beta coefficients estimates based on the whole sample as well as selection rates for each beta based on cross validation iterations, are reported.

Models were calculated with clinical diagnosis as dependent variable as well as with the discrimina- tion of $A \beta_{42} / A \beta_{40}$-ratio positive controls/SCD/MCI cases versus $A \beta_{42} / A \beta_{40}$-ratio negative controls where CSF data were available.

Calculating AUCs for classifying patient groups using hippocampal volumetry used cross-validated unpenalized logistic regression due to lack of collinear covariates.

All model calculations were repeated after adding age, sex, and scanner to assess sensitivity of outcomes to these parameters.

\section{RESULTS}

\section{Demographic data}

Of the 280 included participants, 93 were classified as healthy controls, 9 as cognitively normal first degree relatives of people with AD dementia, 98 as $\mathrm{SCD}, 45$ as MCI, and 35 as AD dementia (see Table 1 for additional participant information). Due to the small number of cases the group of people with first degree relatives with $\mathrm{AD}$ dementia was left out from the subsequent analyses.

The remaining groups differed in respect to gender distribution, age, and years of education (see Table 1 for details on demographic characteristics). As expected and required by diagnostic criteria, $S C D$ and controls did not differ in MMSE scores $(t=1.57$, $189 \mathrm{df}, p=0.12$ ), while MCI and AD cases showed significantly lower MMSE scores compared with controls $(t=7.5,136 \mathrm{df}, p<0.001$, and $t=18.4,126$ df, $p<0.001$, respectively).

CSF was available in 36 controls with 25 having a normal $\mathrm{A} \beta_{42} / \mathrm{A} \beta_{40}$-ratio, in $43 \mathrm{SCD}$ cases with 17 having an abnormal $A \beta_{42} / A \beta_{40}$-ratio, and in $31 \mathrm{MCI}$ cases with 20 having an abnormal $A \beta_{42} / A \beta_{40}$-ratio.

Table 1

Participants' demographic characteristics

\begin{tabular}{lcccc}
\hline N=271 & AD & MCI & SCD & Controls \\
\hline No. cases (women) $^{\mathrm{a}}$ & $35(19)$ & $45(14)$ & $98(47)$ & $93(55)$ \\
Age (SD) [y] $^{\mathrm{b}}$ & $73.5(6.8)$ & $72.3(5.7)$ & $71.3(5.9)$ & $68.5(5.1)$ \\
Education (SD) $^{\mathrm{c}}[\mathrm{y}]$ & $13.4(3.1)$ & $14.4(3.1)$ & $14.6(3.1)$ & $15.1(2.6)$ \\
MMSE (SD) $^{\mathrm{d}}$ & $23.1(3.1)$ & $28.0(1.6)$ & $29.3(0.9)$ & $29.5(0.8)$ \\
\hline
\end{tabular}

${ }^{\mathrm{a}}$ significantly different between groups, $\chi^{2}=9.95,3 \mathrm{df}, p=0.02$;

${ }^{\mathrm{b}}$ significantly different between groups, $\mathrm{F}(3,267)=8.7, p<0.001$;

${ }^{\mathrm{c}}$ significantly different between groups, $\mathrm{F}(3,267)=2.7, p<0.05$;

${ }^{\mathrm{d}}$ significantly different between groups, $\mathrm{F}(3,267)=184.3$, $p<0.001$. 


\section{Voxelwise TBSS analysis}

All models were controlled for age, sex, and scanner as covariates. For SCD versus controls, we did not find significant group differences in FA, MD, nor mode of anisotropy at $p<0.05$ FWE-corrected. Only at an uncorrected $p<0.01$ we found reduced mode of anisotropy in SCD compared with controls in left predominant fornix, fusiform gyrus and superior temporal gyrus white matter, and anterior thalamic radiation (Fig. 1).

For MCI versus controls, we found widespread reductions of FA and increases of MD across limbic and association white matter fiber tracts at $p<0.05$ FWE-corrected (Fig. 2). Reductions in the mode of anisotropy were most focused on the corpus callosum and cingulate gyrus, but also involving external and internal capsule and uncinate fasciculus.

For $\mathrm{AD}$ versus controls, reductions of $\mathrm{FA}$ and increases of MD were similarly widespread as for MCI cases at $p<0.05$, FWE-corrected. Similar to MCI cases, reductions in the mode of anisotropy were focused on the corpus callosum and cingulate gyrus,

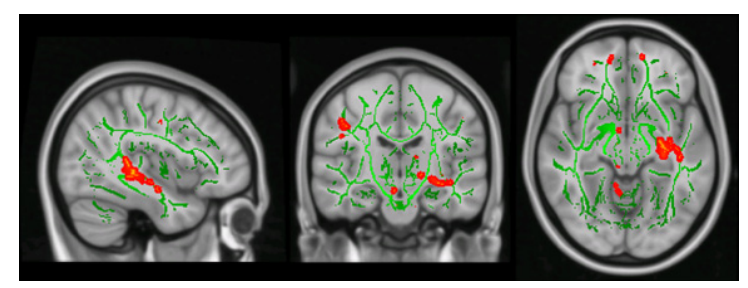

Fig. 1. Differences of mode of anisotropy in SCD cases compared with controls. Projection of the differences of mode of anisotropy values between SCD cases and controls (mode of anisotropy smaller in SCD than in controls) in red to yellow color on the group specific averaged TBSS fiber tract skeleton (green color) in MNI standard space. Effects are thresholded at $p<0.01$, uncorrected for multiple comparisons

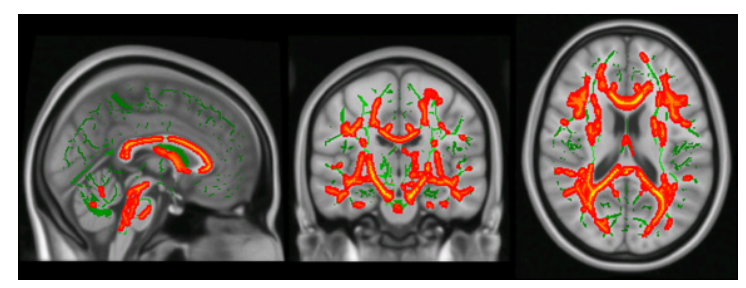

Fig. 2. Differences of FA in in MCI cases compared with controls. Projection of the differences of FA values between MCI cases and controls (FA smaller in MCI than in controls) in red to yellow color on the group specific averaged TBSS fiber tract skeleton (green color) in MNI standard space. Effects are thresholded at $p<0.05$, FWE corrected for multiple comparisons and also involved external and internal capsule and uncinate fasciculus.

In a complementary analysis, we studied tractbased changes of axial and radial diffusivity. We found widespread increase of radial and axial diffusivity in $\mathrm{AD}$ and $\mathrm{MCI}$, but not in SCD, compared with controls at $p<0.05$, FWE-corrected, with a large spatial overlap with the FA decreases (data not shown).

The variance component for scanner was $9.6 \%$ for the mode of anisotropy in the combined clusters discriminating SCD cases and controls. For comparison, the variance component for hippocampus volume in the subsample of SCD and control participants was $1.3 \%$. For the MCI versus controls comparison, the variance component for scanner for the pooled FA clusters was $20.7 \%$, for the MD cluster $29.2 \%$, and for the mode of anisotropy cluster $11.5 \%$; for the hippocampus it was below $1 \%$.

\section{Assessment of diagnostic accuracy}

The variance inflation factor (VIF) for each predictor was calculated as the corresponding diagonal element of the inverse of the cross-correlation matrix [36]. The mean VIF was 44.0 across all DTI parameters and diagnoses. This suggested a high level of collinearity and motivated the use of elastic net logistic regression to account for it.

Mean area under the curve (AUC) with the 95\% confidence intervals for the cross-validated elastic net regression of each group compared to classification via hippocampal volumetry are shown in Figure 3. For the comparison of SCD cases with controls, DTI parameters were numerically superior to hippocampus volume with $69 \%$ versus $62 \%$ AUC. However, for the comparison of $A \beta_{42} / A \beta_{40}$-ratio positive $S C D$ cases (i.e., preclinical $A D$ ) versus $A \beta_{42} / A \beta_{40}$-ratio negative controls, diagnostic accuracy reached only 55\% AUC for the DTI parameters and 60\% AUC for hippocampus volume, respectively. For MCI cases versus controls and for $A \beta_{42} / A \beta_{40}$-ratio positive MCI cases (i.e., prodromal AD) versus $A \beta_{42} / A \beta_{40^{-}}$ ratio negative controls, diagnostic accuracy for DTI parameters was $78 \%$ for both comparisons, and $77 \%$ and $83 \%$ for hippocampus volume, respectively. These numbers were almost unchanged when adding age, sex, and scanner to the classification models.

Table 2 lists important diffusivity measures of specific tract locations that were selected at least in $90 \%$ of the bootstrapped models for classifying

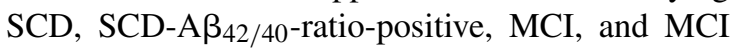

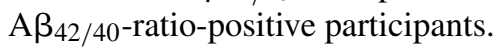




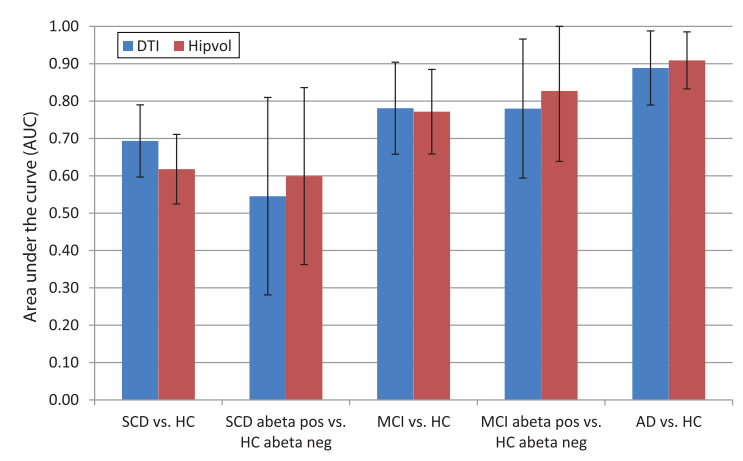

Fig. 3. Group discrimination based on multimodal DTI parameters and hippocampus volume. Cross-validated (100 iterations) areas under the ROC curves (AUC) with 95\% confidence intervals for the group classification of participants of SCD, MCI, and AD versus controls in addition to $\mathrm{MCI} / \mathrm{SCD}$ amyloid- $\beta$ positive versus amyloid- $\beta$ negative controls, based on multimodal tract-based DTI parameters (DTI) and hippocampal volume (Hipvol), respectively.

In an additional analysis, the diagnostic accuracy of DTI parameters comparing $11 \mathrm{~A} \beta_{42 / 40}$-ratiopositive controls plus $17 \mathrm{~A} \beta_{42 / 40}$-ratio-positive SCD cases, representing a preclinical $\mathrm{AD}$ group, versus

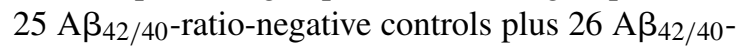
ratio-negative SCD cases, reached a cross-validated AUC of 50\%, i.e., random level accuracy.

\section{DISCUSSION}

Here, we studied diffusion changes as surrogate markers of microstructural lesions in cerebral white matter in clinically defined at risk stages of $\mathrm{AD}$, including SCD and MCI cases, as well as in preclinical and prodromal $\mathrm{AD}$ cases, represented by
CSF amyloid positive cognitively normal people and SCD cases. To assess the potential usefulness of these markers for future diagnostic applications we used a prospective multicenter data set within a cross-validation framework to assess the diagnostic accuracy of diffusion markers in the presence of multicenter variability.

In agreement with previous studies, we found reductions of FA and mode of anisotropy and increases of MD, both in MCI cases in general as well as in A $\beta$-positive MCI cases compared with controls in widespread white matter fiber tracts including projections from hippocampus, as well as association fiber tracts but also parts of the internal capsule, projecting into brain stem white matter. This regional pattern was very similar to previous reports from monocenter studies $[4,5,37-40]$ and the pattern of diffusion changes found in the $\mathrm{AD}$ dementia group of the current cohort. In conclusion, these findings suggest widespread white matter microstructural degeneration already in prodromal stages of $\mathrm{AD}$. The reductions of FA in MCI and $\mathrm{AD}$ cases spatially widely overlapped with increases of radial and axial diffusivity, in agreement with an earlier report in $\mathrm{AD}$ and MCI cases from the ADNI cohort [41]. Consistent with a recent review [42], this would indicate widespread damage including impaired axonal integrity, edema and myelin damage as cause of reduced anisotropy.

Cross-validated diagnostic accuracy of DTI parameters for the distinction of MCI and MCI A $\beta$ positive cases from controls reached almost $80 \%$, but was not superior to hippocampus volume, the bestestablished structural imaging marker of $\mathrm{AD}$ to date.

Table 2

Most frequently selected features for diagnostic group discrimination

\begin{tabular}{|c|c|c|c|c|}
\hline \multicolumn{5}{|c|}{ SCD versus controls } \\
\hline \multirow[t]{2}{*}{$\beta$ coefficient } & \multirow[t]{2}{*}{ Frequency $(\%)$} & \multirow[t]{2}{*}{ Diffusivity measure and region } & \multicolumn{2}{|c|}{$\begin{array}{c}\text { Mean tract diffusivity } \\
\text { value }\end{array}$} \\
\hline & & & Patient & Controls \\
\hline-0.372 & 100 & MO retrolenticular part of internal capsule & 0.225 & 0.233 \\
\hline-0.302 & 96 & MO posterior thalamic radiation $\mathrm{L}$ & 0.217 & 0.227 \\
\hline-0.260 & 93 & MO posterior limb of internal capsule $\mathrm{R}$ & 0.172 & 0.176 \\
\hline-0.237 & 91 & MO fornix/stria terminalis $\mathrm{R}$ & 0.113 & 0.124 \\
\hline-0.239 & 91 & MO uncinate fasciculus L & 0.114 & 0.120 \\
\hline \multicolumn{5}{|c|}{$\mathrm{A} \beta_{42 / 40}$-ratio positive $S C D$ versus $A \beta_{42}$ negative controls } \\
\hline \multicolumn{5}{|c|}{$\begin{array}{c}1 \\
-1\end{array}$} \\
\hline \multicolumn{5}{|c|}{ MCI versus controls } \\
\hline-0.023 & 100 & MO medial lemniscus $\mathrm{R}$ & 0.207 & 0.219 \\
\hline \multicolumn{5}{|c|}{$\mathrm{A} \beta_{42 / 40}$-ratio positive MCI versus $A \beta_{42}$ negative controls } \\
\hline & & $-^{2}$ & & \\
\hline
\end{tabular}

${ }^{1}$ Highest selection frequency was $71 \% ;{ }^{2}$ highest selection frequency was $88 \%$. 
This agrees with the level of accuracy found in previous monocenter studies $[4,5]$, including studies using tract-based statistics [43]. Interestingly, the elastic net algorithm selected only few fiber tracts with very high frequency $(>90 \%)$, another set of association fiber tracts had between $50 \%$ to $80 \%$ selection frequency. This is consistent with the widespread alterations of white matter fiber tracts, suggesting comparable diagnostic value of a wide range of tracts. The mode of anisotropy is a scalar diffusion marker that describes an important aspect of the shape of the diffusion tensor. It ranges between -1 and 1 as the shape of the diffusion tensor ranges from planar anisotropy (in areas with crossing fiber populations or adjacent orthogonal fiber orientations) through orthotropy to linear anisotropy (in areas with one predominant fiber direction) [44]. In a study using joint independent component analysis, the mode of anisotropy was decreased in MCI subjects compared to controls mainly in anterior and posterior corpus callosum and in superior and inferior longitudinal fasciculus [45]. Consistently, in the current analysis we found reductions of the mode of anisotropy in MCI in corpus callosum and cingulate gyrus, but also involving external and internal capsule and uncinate fasciculus. Thus, the mode of anisotropy reductions involved mainly regions with directed fiber tracts such as corpus callosum or cingulate bundle, indicating a loss of these directed fibers, resulting in a more orthotropic or planar shape of the diffusion tensor.

SCD cases exhibited no significant fiber tract alterations at family wise error corrected $p$-values. Only at a liberal uncorrected level of significance of $p<0.01$, we found reductions in the mode of anisotropy in restricted white matter regions, including left predominant medial temporal lobe projections. This agrees with previous monocenters studies, where diffusion parameter changes were found in some [13, 16, 46-48], but not all studies [49, 50], and one of the positive studies did not apply a correction for multiple comparisons [13]. In addition, none of these previous studies included amyloid markers to assess preclinical AD status of the SCD cases. The inconsistency of results across studies may reflect an only low to moderate effect size of diffusion parameter changes in SCD cases. Mode of anisotropy changes in SCD cases compared with controls have not been reported before, rendering a comparison of our findings with previous results unfeasible. The only previous study on mode of anisotropy changes in SCD used the SCD group as reference group for comparison with $\mathrm{AD}$ and MCI [15].
The likelihood of false positive findings was high in the analysis that was uncorrected for multiple comparisons. Still, it is interesting to discuss why the mode of anisotropy, indicating a change in the diffusion tensor toward a more sphere like shape, may be the earliest diffusion marker affected in SCD in our analysis. One possible reason may be that among the three diffusion markers studied, the mode of anisotropy was least affected by multicenter effects, with a variance component of about $10 \%$ attributable to scanner, as compared with $20 \%$ for FA and almost $30 \%$ for MD. In addition, one could speculate that the reduction of the mode of anisotropy indicates a selective loss of highly directional fiber tracts within particularly vulnerable regions, such as fornix and temporal lobe white matter, but this needs to be confirmed in subsequent studies.

Diagnostic accuracy for the discrimination of SCD cases from healthy controls was only moderate to low with $69 \%$, and mainly driven by reductions of mode of anisotropy in the uncinate fascicle, fornix and retrolenticular part of internal capsule, but substantially higher than for hippocampus volume with $62 \%$. This compares with an AUC of $78 \%$ for the discrimination between 20 SCD cases and 22 controls in a previous study that did, however, not use cross-validation and therefore strongly overestimated the level of accuracy [16].

When comparing A $\beta$-positive SCD cases, representing a preclinical stage of $\mathrm{AD}$, with $\mathrm{A} \beta$-negative controls, DTI parameters only reached random level guessing accuracy. The same was true for the comparison of the $A \beta$-positive and $A \beta$-negative controls and the combined analysis of $A \beta$-positive SCD and controls versus the A $\beta$-negative SCD cases and controls. The latter analysis included 28 amyloid positive and 51 amyloid negative cases; this substantial number of cases suggests that the lack of an effect is not just a false negative outcome. In conclusion, the white matter alterations in the SCD cases (found at an uncorrected level of significance) may be related to the clinical phenotype rather than the underlying amyloid pathology. SCD is an unspecific syndrome related to "numerous conditions such as normal aging, personality traits, psychiatric conditions, neurologic and medical disorders, substance use, and medication. It may also be affected by the individual cultural background." ([10], pages 845/646). Thus, the white matter alterations in the SCD phenotype cases may reflect a trait from a broad range of conditions which are independent from the state of $\mathrm{A} \beta$ pathology. Consistently, not all cases with SCD 
progress to MCI [11] and not all MCI cases have transited through a state of SCD [51]. This would suggest that similar to white matter changes preceding the first episode of major depression [52], the white matter alterations in SCD cases may reflect a functional (and in some cases reversible) clinical syndrome, rather than the effect from neuropathological lesions. The DELCODE protocol excludes current or past episodes of major depression as well as significant cerebrovascular disease. However, subsyndromal depressive symptoms or subclinical personality traits, such as increased anxiety, that may be related to subtle white matter alterations were not excluded.

There are several limitations associated with this study. First, the number of cases with available CSF was smaller than one would have wished for. Still, to our knowledge, this is the first DTI study featuring a substantial number of SCD cases stratified according to their amyloid status. Secondly, multicenter variability affects the accuracy of diffusion markers. This was even true for the prospectively harmonized DTI data acquisition and the use of tract bases spatial statistic (that was found less sensitive to multicenter effects than voxel-based analysis in a clinical phantom study [8]). Multicenter acquisition, however, is required if one wants to test the useful of DTI measures for the application in future (multicenter) clinical trials or routine care. The variance component analysis revealed that between $9 \%$ and $29 \%$ of variance was attributable to scanner for the DTI parameters. This compares favorably with previous analysis on multicenter DTI parameters from retrospectively pooled data with more than $40 \%$ of variance attributable to scanner for FA and MD parameters in a voxel-based analysis [53]. But even mode of anisotropy that was the least affected by scanner effects among the diffusion parameters was still much more affected than hippocampus volume. Other shortcomings are the different age and sex distribution across the diagnostic groups. However, including these variables together with scanner did not affect the outcome of the diagnostic models in sensitivity analyses.

In conclusion, we found significant differences in widespread white matter tracts in MCI and AD cases compared with controls, including $\mathrm{A} \beta$ positive $\mathrm{MCI}$ cases, representing prodromal AD. In contrast, white matter alterations were detectable only at an uncorrected level of significance and spatially restricted in the SCD cases and were entirely absent in A $\beta$ positive compared with $A \beta$ negative SCD and control cases, suggesting an effect of clinical phenotype of $S C D$ rather than of preclinical $A \beta$ pathology on white matter tract integrity in this cohort. In the near future, we will have access to the longitudinal data of the DELCODE cohort allowing assessment of the predictive accuracy of DTI parameters for cognitive decline within the $\mathrm{AD}$ spectrum.

\section{DISCLOSURE STATEMENT}

Authors' disclosures available online (https:// www.j-alz.com/manuscript-disclosures/19-0446r1).

\section{REFERENCES}

[1] Caso F, Agosta F, Filippi M (2016) Insights into white matter damage in Alzheimer's disease: From postmortem to in vivo diffusion tensor MRI studies. Neurodegener Dis 16, 26-33.

[2] Le Bihan D, Mangin JF, Poupon C, Clark CA, Pappata S, Molko N, Chabriat H (2001) Diffusion tensor imaging: Concepts and applications. J Magn Reson Imaging 13, 534-546.

[3] Moore EE, Hohman TJ, Badami FS, Pechman KR, Osborn KE, Acosta LMY, Bell SP, Babicz MA, Gifford KA, Anderson AW, Goldstein LE, Blennow K, Zetterberg H, Jefferson AL (2018) Neurofilament relates to white matter microstructure in older adults. Neurobiol Aging 70, 233241.

[4] Cui Y, Wen W, Lipnicki DM, Beg MF, Jin JS, Luo S, Zhu W, Kochan NA, Reppermund S, Zhuang L, Raamana PR, Liu T, Trollor JN, Wang L, Brodaty H, Sachdev PS (2012) Automated detection of amnestic mild cognitive impairment in community-dwelling elderly adults: A combined spatial atrophy and white matter alteration approach. Neuroimage 59, 1209-1217.

[5] Henf J, Grothe MJ, Brueggen K, Teipel S, Dyrba M (2018) Mean diffusivity in cortical gray matter in Alzheimer's disease: The importance of partial volume correction. $\mathrm{Neu}$ roimage Clin 17, 579-586.

[6] Brueggen K, Grothe MJ, Dyrba M, Fellgiebel A, Fischer F, Filippi M, Agosta F, Nestor P, Meisenzahl E, Blautzik J, Frolich L, Hausner L, Bokde ALW, Frisoni G, Pievani M, Kloppel S, Prvulovic D, Barkhof F, Pouwels PJW, Schroder J, Hampel H, Hauenstein K, Teipel S (2017) The European DTI Study on Dementia - A multicenter DTI and MRI study on Alzheimer's disease and Mild Cognitive Impairment. Neuroimage 144, 305-308.

[7] Dyrba M, Barkhof F, Fellgiebel A, Filippi M, Hausner L, Hauenstein K, Kirste T, Teipel SJ; EDSD study group (2015) Predicting prodromal Alzheimer's disease in subjects with mild cognitive impairment using machine learning classification of multimodal multicenter diffusion-tensor and magnetic resonance imaging data. J Neuroimaging $\mathbf{2 5}$, 738747.

[8] Teipel SJ, Reuter S, Stieltjes B, Acosta-Cabronero J, Ernemann U, Fellgiebel A, Filippi M, Frisoni G, Hentschel F, Jessen F, Kloppel S, Meindl T, Pouwels PJW, Hauenstein KH, Hampel H (2011) Multicenter stability of diffusion tensor imaging measures: A European clinical and physical phantom study. Psychiatry Res 194, 363-371.

[9] Frisoni GB, Boccardi M, Barkhof F, Blennow K, Cappa S, Chiotis K, Demonet JF, Garibotto V, Giannakopoulos 
P, Gietl A, Hansson O, Herholz K, Jack CR, Jr., Nobili F, Nordberg A, Snyder HM, Ten Kate M, Varrone A, Albanese E, Becker S, Bossuyt P, Carrillo MC, Cerami C, Dubois B, Gallo V, Giacobini E, Gold G, Hurst S, Lonneborg A, Lovblad KO, Mattsson N, Molinuevo JL, Monsch AU, Mosimann U, Padovani A, Picco A, Porteri C, Ratib O, Saint-Aubert L, Scerri C, Scheltens P, Schott JM, Sonni I, Teipel S, Vineis P, Visser PJ, Yasui Y, Winblad B (2017) Strategic roadmap for an early diagnosis of Alzheimer's disease based on biomarkers. Lancet Neurol 16, 661-676.

[10] Jessen F, Amariglio RE, van Boxtel M, Breteler M, Ceccaldi M, Chetelat G, Dubois B, Dufouil C, Ellis KA, van der Flier WM, Glodzik L, van Harten AC, de Leon MJ, McHugh P, Mielke MM, Molinuevo JL, Mosconi L, Osorio RS, Perrotin A, Petersen RC, Rabin LA, Rami L, Reisberg B, Rentz DM, Sachdev PS, de la Sayette V, Saykin AJ, Scheltens P, Shulman MB, Slavin MJ, Sperling RA, Stewart R, Uspenskaya O, Vellas B, Visser PJ, Wagner M, Subjective Cognitive Decline Initiative Working Group (2014) A conceptual framework for research on subjective cognitive decline in preclinical Alzheimer's disease. Alzheimers Dement 10, 844-852.

[11] Mitchell AJ, Beaumont H, Ferguson D, Yadegarfar M, Stubbs B (2014) Risk of dementia and mild cognitive impairment in older people with subjective memory complaints: Meta-analysis. Acta Psychiatr Scand 130, 439-451.

[12] Hong YJ, Kim CM, Jang EH, Hwang J, Roh JH, Lee JH (2016) White matter changes may precede gray matter loss in elderly with subjective memory impairment. Dement Geriatr Cogn Disord 42, 227-235.

[13] Hong YJ, Yoon B, Shim YS, Ahn KJ, Yang DW, Lee JH (2015) Gray and white matter degenerations in subjective memory impairment: Comparisons with normal controls and mild cognitive impairment. J Korean Med Sci 30, 16521658.

[14] Selnes P, Aarsland D, Bjornerud A, Gjerstad L, Wallin A, Hessen E, Reinvang I, Grambaite R, Auning E, Kjaervik VK, Due-Tonnessen P, Stenset V, Fladby T (2013) Diffusion tensor imaging surpasses cerebrospinal fluid as predictor of cognitive decline and medial temporal lobe atrophy in subjective cognitive impairment and mild cognitive impairment. J Alzheimers Dis 33, 723-736.

[15] Doan NT, Engvig A, Persson K, Alnaes D, Kaufmann T, Rokicki J, Cordova-Palomera A, Moberget T, Braekhus A, Barca ML, Engedal K, Andreassen OA, Selbaek G, Westlye LT (2017) Dissociable diffusion MRI patterns of white matter microstructure and connectivity in Alzheimer's disease spectrum. Sci Rep 7, 45131.

[16] Shao W, Li X, Zhang J, Yang C, Tao W, Zhang S, Zhang Z, Peng D (2019) White matter integrity disruption in the predementia stages of Alzheimer's disease: From subjective memory impairment to amnestic mild cognitive impairment. Eur J Neurol 26, 800-807.

[17] Jack CR, Jr., Bennett DA, Blennow K, Carrillo MC, Dunn B, Haeberlein SB, Holtzman DM, Jagust W, Jessen F, Karlawish J, Liu E, Molinuevo JL, Montine T, Phelps C, Rankin KP, Rowe CC, Scheltens P, Siemers E, Snyder HM, Sperling R, Contributors (2018) NIA-AA Research Framework: Toward a biological definition of Alzheimer's disease. Alzheimers Dement 14, 535-562.

[18] Jessen F, Spottke A, Boecker H, Brosseron F, Buerger K, Catak C, Fliessbach K, Franke C, Fuentes M, Heneka MT, Janowitz D, Kilimann I, Laske C, Menne F, Nestor P, Peters O, Priller J, Pross V, Ramirez A, Schneider A, Speck O, Spruth EJ, Teipel S, Vukovich R, Westerteicher C, Wiltfang
J, Wolfsgruber S, Wagner M, Duzel E (2018) Design and first baseline data of the DZNE multicenter observational study on predementia Alzheimer's disease (DELCODE). Alzheimers Res Ther 10, 15.

[19] Gauggel S, Birkner B (1999) Validity and reliability of a German version of the Geriatric Depression Scale (GDS). Z Klin Psychol Forsch Praxis 28, 18-27.

[20] Albert MS, DeKosky ST, Dickson D, Dubois B, Feldman HH, Fox NC, Gamst A, Holtzman DM, Jagust WJ, Petersen RC, Snyder PJ, Carrillo MC, Thies B, Phelps CH (2011) The diagnosis of mild cognitive impairment due to Alzheimer's disease: Recommendations from the National Institute on Aging-Alzheimer's Association workgroups on diagnostic guidelines for Alzheimer's disease. Alzheimers Dement 7, 270-279.

[21] McKhann GM, Knopman DS, Chertkow H, Hyman BT, Jack CR, Jr., Kawas CH, Klunk WE, Koroshetz WJ, Manly JJ, Mayeux R, Mohs RC, Morris JC, Rossor MN, Scheltens P, Carrillo MC, Thies B, Weintraub S, Phelps $\mathrm{CH}$ (2011) The diagnosis of dementia due to Alzheimer's disease: Recommendations from the National Institute on Aging-Alzheimer's Association workgroups on diagnostic guidelines for Alzheimer's disease. Alzheimers Dement 7, 263-269.

[22] Acosta-Cabronero J, Williams GB, Pengas G, Nestor PJ (2010) Absolute diffusivities define the landscape of white matter degeneration in Alzheimer's disease. Brain 133, 529539.

[23] Oishi K, Mielke MM, Albert M, Lyketsos CG, Mori S (2011) DTI analyses and clinical applications in Alzheimer's disease. J Alzheimers Dis 26(Suppl 3), 287-296.

[24] Stricker NH, Schweinsburg BC, Delano-Wood L, Wierenga CE, Bangen KJ, Haaland KY, Frank LR, Salmon DP, Bondi MW (2009) Decreased white matter integrity in latemyelinating fiber pathways in Alzheimer's disease supports retrogenesis. Neuroimage 45, 10-16.

[25] Smith SM, Jenkinson M, Johansen-Berg H, Rueckert D, Nichols TE, Mackay CE, Watkins KE, Ciccarelli O, Cader MZ, Matthews PM, Behrens TE (2006) Tract-based spatial statistics: Voxelwise analysis of multi-subject diffusion data. Neuroimage 31, 1487-1505.

[26] Jenkinson M, Bannister P, Brady M, Smith S (2002) Improved optimization for the robust and accurate linear registration and motion correction of brain images. $\mathrm{Neu}$ roimage $17,825-841$.

[27] Smith S (2002) Fast robust automated brain extraction. Hum Brain Mapp 17, 143-155.

[28] Dubois B, Feldman HH, Jacova C, Dekosky ST, BarbergerGateau P, Cummings J, Delacourte A, Galasko D, Gauthier S, Jicha G, Meguro K, O'Brien J, Pasquier F, Robert P, Rossor M, Salloway S, Stern Y, Visser PJ, Scheltens P (2007) Research criteria for the diagnosis of Alzheimer's disease: Revising the NINCDS-ADRDA criteria. Lancet Neurol 6, 734-746.

[29] Frisoni GB, Jack CR, Jr., Bocchetta M, Bauer C, Frederiksen KS, Liu Y, Preboske G, Swihart T, Blair M, Cavedo E, Grothe MJ, Lanfredi M, Martinez O, Nishikawa M, Portegies M, Stoub T, Ward C, Apostolova LG, Ganzola R, Wolf D, Barkhof F, Bartzokis G, DeCarli C, Csernansky JG, deToledo-Morrell L, Geerlings MI, Kaye J, Killiany RJ, Lehericy S, Matsuda H, O'Brien J, Silbert LC, Scheltens P, Soininen H, Teipel S, Waldemar G, Fellgiebel A, Barnes J, Firbank M, Gerritsen L, Henneman W, Malykhin N, Pruessner JC, Wang L, Watson C, Wolf $\mathrm{H}$, deLeon M, Pantel J, Ferrari C, Bosco P, Pasqualetti P, Duchesne S, Duvernoy 
H, Boccardi M, EADC-ADNI Working Group on The Harmonized Protocol for Manual Hippocampal Volumetry and for the Alzheimer's Disease Neuroimaging Initiative (2015) The EADC-ADNI Harmonized Protocol for manual hippocampal segmentation on magnetic resonance: Evidence of validity. Alzheimers Dement 11, 111-125.

[30] Wolf D, Bocchetta M, Preboske GM, Boccardi M, Grothe MJ, Alzheimer's Disease Neuroimaging Initiative (2017) Reference standard space hippocampus labels according to the European Alzheimer's Disease Consortium-Alzheimer's Disease Neuroimaging Initiative harmonized protocol: Utility in automated volumetry. Alzheimers Dement 13, 893-902.

[31] Ashburner J (2007) A fast diffeomorphic image registration algorithm. Neuroimage 38, 95-113.

[32] Janelidze S, Zetterberg H, Mattsson N, Palmqvist S, Vanderstichele H, Lindberg O, van Westen D, Stomrud E, Minthon L, Blennow K, Swedish BioFINDER study group, Hansson O (2016) CSF Abeta42/Abeta40 and Abeta42/Abeta38 ratios: Better diagnostic markers of Alzheimer disease. Ann Clin Transl Neurol 3, 154-165.

[33] Zou H, Hastie T (2005) Regularization and variable selection via the elastic net. J R Stat Soc Series B Stat Methodol 67, 301-320.

[34] Teipel SJ, Grothe MJ, Metzger CD, Grimmer T, Sorg C, Ewers M, Franzmeier N, Meisenzahl E, Kloppel S, Borchardt V, Walter M, Dyrba M (2016) Robust detection of impaired resting state functional connectivity networks in Alzheimer's disease using elastic net regularized regression. Front Aging Neurosci 8, 318.

[35] Mori S, Oishi K, Jiang H, Jiang L, Li X, Akhter K, Hua K, Faria AV, Mahmood A, Woods R, Toga AW, Pike GB, Neto PR, Evans A, Zhang J, Huang H, Miller MI, van Zijl P, Mazziotta J (2008) Stereotaxic white matter atlas based on diffusion tensor imaging in an ICBM template. Neuroimage 40, 570-582.

[36] Belsley DA (1991) Conditioning Diagnostics: Collinearity and Weak Data in Regression, John Wiley \& Sons, Chichester.

[37] Cho H, Yang DW, Shon YM, Kim BS, Kim YI, Choi YB, Lee KS, Shim YS, Yoon B, Kim W, Ahn KJ (2008) Abnormal integrity of corticocortical tracts in mild cognitive impairment: A diffusion tensor imaging study. J Korean Med Sci 23, 477-483.

[38] Alves GS, O'Dwyer L, Jurcoane A, Oertel-Knochel V, Knochel C, Prvulovic D, Sudo F, Alves CE, Valente L, Moreira D, Fusser F, Karakaya T, Pantel J, Engelhardt E, Laks J (2012) Different patterns of white matter degeneration using multiple diffusion indices and volumetric data in mild cognitive impairment and Alzheimer patients. PLoS One 7, e52859.

[39] Clerx L, Visser PJ, Verhey F, Aalten P (2012) New MRI markers for Alzheimer's disease: A meta-analysis of diffusion tensor imaging and a comparison with medial temporal lobe measurements. J Alzheimers Dis 29, 405-429.

[40] Liu Y, Spulber G, Lehtimaki KK, Kononen M, Hallikainen I, Grohn H, Kivipelto M, Hallikainen M, Vanninen R, Soininen $H$ (2011) Diffusion tensor imaging and tract-based spatial statistics in Alzheimer's disease and mild cognitive impairment. Neurobiol Aging 32, 1558-1571.

[41] Lee SH, Coutu JP, Wilkens P, Yendiki A, Rosas HD, Salat DH, Alzheimer's disease Neuroimaging Initiative
(2015) Tract-based analysis of white matter degeneration in Alzheimer's disease. Neuroscience 301, 79-89.

[42] Winklewski PJ, Sabisz A, Naumczyk P, Jodzio K, Szurowska E, Szarmach A (2018) Understanding the physiopathology behind axial and radial diffusivity changes-what do we know? Front Neurol 9, 92.

[43] Zhuang L, Wen W, Zhu W, Trollor J, Kochan N, Crawford J, Reppermund S, Brodaty H, Sachdev P (2010) White matter integrity in mild cognitive impairment: A tract-based spatial statistics study. Neuroimage 53, 16-25.

[44] Ennis DB, Kindlmann G (2006) Orthogonal tensor invariants and the analysis of diffusion tensor magnetic resonance images. Magn Reson Med 55, 136-146.

[45] Teipel SJ, Grothe MJ, Filippi M, Fellgiebel A, Dyrba M, Frisoni GB, Meindl T, Bokde AL, Hampel H, Kloppel S, Hauenstein K; EDSD study group (2014) Fractional anisotropy changes in Alzheimer's disease depend on the underlying fiber tract architecture: A multiparametric DTI study using joint independent component analysis. $J$ Alzheimers Dis 41, 69-83.

[46] Li XY, Tang ZC, Sun Y, Tian J, Liu ZY, Han Y (2016) White matter degeneration in subjective cognitive decline: A diffusion tensor imaging study. Oncotarget 7, 54405-54414.

[47] Ryu SY, Lim EY, Na S, Shim YS, Cho JH, Yoon B, Hong YJ, Yang DW (2017) Hippocampal and entorhinal structures in subjective memory impairment: A combined MRI volumetric and DTI study. Int Psychogeriatr 29, 785-792.

[48] Selnes P, Fjell AM, Gjerstad L, Bjornerud A, Wallin A, Due-Tonnessen P, Grambaite R, Stenset V, Fladby T (2012) White matter imaging changes in subjective and mild cognitive impairment. Alzheimers Dement 8, S112-121.

[49] Kiuchi K, Kitamura S, Taoka T, Yasuno F, Tanimura M, Matsuoka K, Ikawa D, Toritsuka M, Hashimoto K, Makinodan M, Kosaka J, Morikawa M, Kichikawa K, Kishimoto $\mathrm{T}$ (2014) Gray and white matter changes in subjective cognitive impairment, amnestic mild cognitive impairment and Alzheimer's disease: A voxel-based analysis study. PLoS One 9, e104007.

[50] Wang Y, West JD, Flashman LA, Wishart HA, Santulli RB, Rabin LA, Pare N, Arfanakis K, Saykin AJ (2012) Selective changes in white matter integrity in MCI and older adults with cognitive complaints. Biochim Biophys Acta 1822, 423-430.

[51] Sargent-Cox K, Cherbuin N, Sachdev P, Anstey KJ (2011) Subjective health and memory predictors of mild cognitive disorders and cognitive decline in ageing: The Personality and Total Health (PATH) through Life Study. Dement Geriatr Cogn Disord 31, 45-52.

[52] Chen G, Guo Y, Zhu H, Kuang W, Bi F, Ai H, Gu Z, Huang X, Lui S, Gong Q (2017) Intrinsic disruption of white matter microarchitecture in first-episode, drug-naive major depressive disorder: A voxel-based meta-analysis of diffusion tensor imaging. Prog Neuropsychopharmacol Biol Psychiatry 76, 179-187.

[53] Teipel SJ, Wegrzyn M, Meindl T, Frisoni G, Bokde AL, Fellgiebel A, Filippi M, Hampel H, Kloppel S, Hauenstein K, Ewers M (2012) Anatomical MRI and DTI in the diagnosis of Alzheimer's disease: A European multicenter study. J Alzheimers Dis 31(Suppl 3), S33-47. 